\title{
Improving treatment provision of Wet AMD with intravitreal ranibizumab
}

\author{
Fadi Ghazala, Marta Hovan, Sajjad Mahmood
}

\begin{abstract}
Wet age-related macular degeneration (AMD) treatment using intravitreal ranibizumab needs to be started as soon as possible and treatment must be administered based on regular review to achieve the best results. In clinical practice this tight schedule is a challenge and methods of carrying out such timely treatment is the objective of this quality improvement work. A departmental audit was carried out on the service providing treatment for patients with wet AMD in 2009. This audit identified that the appointment system did not meet the ideal standards and subsequently wet AMD patients' visual outcome were poorer than the standard set in trials where the patients were seen and treated at predetermined intervals. In order to enhance the visual benefit of the administered therapy for our patients we thought it necessary to find ways to see and treat patients sooner as well as reduce time intervals between follow-up appointments. The quality improvement was carried out through redesigning the service in the macular treatment centre of Manchester Royal Eye Hospital. Three main strategies were implemented including: changes to the appointment system, expansion of the treatment facility and employment of additional staff. Following changes made, regular re-audits were used to analyse the effectiveness of the new strategies. The changes introduced have brought appointment standards to the level of Royal College of Ophthalmologists' recommendations. Consequently visual outcomes were approaching the standards set by landmark studies. The visual improvement of treated patients seen in the 2011 audit are comparable to other reports outside clinical trials in the UK. In order to enhance the efficacy of ranibizumab for wet AMD it is essential for treatment to be initiated as soon as possible and administered to patients at the recommended time intervals. The actions we have taken were effective in helping develop a service performing to higher standards.
\end{abstract}

\section{Problem}

Age-related macular degeneration (AMD) is the most common cause of visual impairment in adults over the age of 50 in the developed world. This condition is a major health concern with over 250,000 adults in the United Kingdom suffering from blindness due to AMD.(1) A decade ago treatment options were limited with doctors playing more of a supportive role in the care of these patients. The development of anti-VEGF therapies acting against vascular endothelial growth factor (VEGF) thus limiting inappropriate new vessel formation has revolutionized the treatment of wet AMD. Ranibizumab (Lucentis $\AA$; Novartis Pharma AG, Basel, Switzerland; Genentech Inc., South San Francisco, CA, USA) is an effective anti-VEGF drug licensed for use in wet AMD.(2) However, if patients are not seen and do not receive therapy at the recommended time schedule their visual outcomes are reported to be inferior as evidenced by landmark studies.(3) With an ageing population and growing numbers of patients to treat, administration of ranibizumab as dictated by the Royal College of

Ophthalmologists is particularly challenging. In the largest cohort study to date investigating compliance to these standards in France, not one patient (of 551 patients followed by 16 ophthalmologists) adhered to the treatment schedule entirely.(4) These guidelines recommend ranibizumab treatment be started within 2 weeks from referral to the eye unit and within 1 week from the first hospital visit.(5) In what is referred to as the loading phase of treatment, the first 3 injections must be given at monthly intervals. Further ranibizumab injections need to be administered as indicated guided by regular monthly reviews in the maintenance phase of treatment.(5) This treatment regimen places strain on treatment centres as there are limited resources and an ever accumulating cohort of patients. None-the-less, its importance cannot be overlooked as poorer outcomes have been demonstrated where initiation of treatment was delayed(6) and where review intervals have been longer. Further, it has been demonstrated that better baseline vision at the start of treatment (i.e. diagnosing and treating patients before there is a significant deterioration in vision) results in better vision following treatment.(7)

\section{Background}

This quality improvement work was carried out in Manchester Royal Eye Hospital's macular treatment centre (MTC) where AMD patients have been assessed and treated with ranibizumab intravitreal injections since 2008. The MTC is not only dedicated to the management of wet macular degeneration but also macular oedema secondary to other eye conditions (e.g. retinal vein occlusions and diabetic retinopathy). When a patient is suspected to have wet AMD they are referred to the macular treatment centre. An appointment is made for the patient to be assessed and considered for ranibizumab treatment. On each appointment day images are taken by a qualified technician. The patient is then seen by a doctor who decides if treatment is appropriate and if it is, the patient receives an intravitreal ranibizumab injection. In 2008 when anti-VEGF treatment started the MTC consisted of: 1 room to assess vision, 3 consultation rooms, 2 imaging suites -one for ocular coherence tomography (OCT) to quantify macular thickness and one for fluorescein angiography (FFA). The treatment room has positive pressure ventilation and exceeds Royal College of Ophthalmologists' guidance. The employed healthcare workers included: 3 consultant ophthalmologists with interest in retinal diseases, 2 ophthalmic fellows, 1 specialist nurse, 1 optometrist and an imaging technician. Outside its role as a regional treatment centre, the MTC facility is also used extensively for multicentre trials 
and other research projects. Continuous audits have been used to improve the AMD service offered at the MTC thus improving treatment response in patients' vision. In 2009 an audit identified that appointment standards were not being met and visual acuity measures fell short in comparison to landmark studies. In order to see greater improvement in the visual acuity of patients with wet AMD it was necessary to find ways to organise appointments for new patients sooner as well as reduce time intervals between followup visits. The aim of this article is to identify the changes to service management that have been brought to action based on the reports of repeated audits since 2009. Our project aimed to identify shortcomings of the appointment system and find ways to rectify them. Following the changes made to the appointment system, MTC facility, and staffing we showed an improvement in following the recommended treatment schedule that translated into an improvement in patients' visual outcomes.

\section{MEASURES FOR IMPROVEMENT}

The main aim of this project was to improve treatment provision of wet AMD patients by treating according to Royal College of Opthalmologists' guidelines.(5) Namely, reduce time to first assessment, first treatment and time intervals between subsequent treatments. Ultimately, the key measure of improvement was visual acuity of patients treated for wet AMD.

\section{Baseline Measurement}

A retrospective audit carried out on the service in 2009 showed that appointment standards were not being met and visual acuity measures fell short in comparison to landmark studies. This audit consisted of two arms. The first arm was an audit of the case notes of 100 consecutive patients attending the AMD clinic and examined the referral system. The second arm of the study audited 62 patients who had at least 1 year follow-up and focussed on the follow-up period and the visual results.

The re-audit of 2010 was a prospective case note review of 53 randomly selected patients whose notes were available for audit.

The subsequent re-audit in 2011 was also a prospective case note review of 60 consecutive follow-up patients attending MTC examining the follow-up system and 20 new patients that focussed on how soon initial assessment was taking place after referral to the eye clinic.

The following measures were recorded:

- Time from referral to initial treatment

- Time intervals between visits during the first year of treatment

- Visual outcomes achieved at 1st visit after 3rd injection and 12 months from 1 st injection

- Mean visual acuity change in ETDRS letters - measured on the chart used by the Early Treatment for Diabetic Retinopathy Study
- Number of patients who gained 15 ETDRS letters or more - at first visit after 3rd injection, 4 and 12 months after first injection

- Number of patients losing less than 15 ETDRS letters - at 4 and 12 months following first injection

- Number of visits during the first treatment year

- Number of injections required during this interval and

- Documented complications

This data was analysed to compare our practice to the chosen standards.

\section{OUTCOME MEASURES}

Two types of standards were used for appraisal:

1. standards related to the initiation of first assessment and review intervals (appointment standards)

2. standards related to the expected outcome of treatment (visual outcome standards)

The appointment standards were based on the Royal College of Ophthalmologists' recommendations:

a. Time from referral to 1 st assessment needs be within one week and from 1st clinic appointment to treatment can be another 1 week. This means that patients who need treatment ideally receive their first ranibizumab injection within 2 weeks from referral.

b. Treatment should be initially administered as 3 injections at 3-monthly intervals (loading phase)

c. After loading phase, monthly review should continue to guide treatment (maintenance phase)

Visual outcome standards chosen as benchmark were from the landmark studies listed in the NICE guidelines. These include ANCHOR, MARINA, PIER and PRONTO. (8-11)

ANCHOR: randomized, double-masked, study of ranibizumab (Lucentis) administered as a monthly intravitreal injection compared with photodynamic therapy in patients with classic subfoveal neovascular membrane secondary to AMD. One year results showed that $94 \%$ or more of ranibizumab-treated patients maintained vision (lost less than 15 ETDRS letters) compared to $64 \%$ of patients receiving photodynamic therapy. Up to $40 \%$ of ranibizumab treated patients improved vision (gained 15 or more ETDRS letters) compared to $6 \%$ of patients receiving photodynamic therapy.(8)

MARINA: randomized, double-masked, sham injection-controlled study of the efficacy and safety of ranibizumab injected monthly in patients with minimally classic or occult subfoveal neovascular membrane due to wet AMD. Two-year results show that $90 \%$ or more of ranibizumab-treated patients maintained vision compared 
to $53 \%$ of those in the control group. Up to $33 \%$ of ranibizumabtreated patients improved vision compared to $4 \%$ of the control group.(9)

PIER: study evaluated whether three monthly injections of ranibizumab as loading phase and then injections every three months was as effective to maintain and improve vision as the previous studies. After twelve months of follow-up, treated patients had the same vision as they did at baseline, with no significant improvement documented.(10)

PRONTO: study showed that the visual improvement observed after three injections of loading phase treatment may be maintained if patients are reviewed monthly and receive treatment pro re nata (prn) guided by changes in visual acuity and retinal thickness on OCT.(11)

See supplementary file: ds1905.docx - "Results"

\section{Design}

The study design was audit of patient treatment times and visual acuity measures and continuous re-audit to measure the impact of the actions taken. Through regular re-audit it was possible to measure the effects of changes made at the MTC on treatment times and the corresponding effect on the mean visual acuity.

A departmental audit was carried out on the AMD service in 2009. This audit identified that the appointment system did not meet standards and wet AMD patients' visual outcome was poorer than the standard set in trials where the patients were seen and treated at predetermined intervals. In order to enhance the visual benefit of the administered therapy we found ways to see and treat patients sooner as well as reduce time intervals between follow-up appointments.

The quality improvement was carried out through redesigning the service in the MTC. Following the changes made, regular re-audits were used to analyze the effectiveness of the new strategies.

\section{Strategy}

In order to see greater improvement in the mean visual acuity of patients with wet AMD we continuously aimed to find ways to see and treat patients according to the guidelines.

Following the first audit several changes aimed at improving vision were made: some by reducing appointment/treatment intervals, others by employing more staff and streamlining the assessment and treatment process.

In order to improve appointment standards and visual outcome standards the following action plans were carried out between 2009-2011:

- Fast track referral pathway into hospital eye service for wet AMD patients was implemented
- Application process for funding of ranibizumab injections from primary care trust was streamlined so that no prior approval was required before commencing treatment

- With the agreement of hospital management, proposed changes to clinic templates were made and new protected slots became available for new patients to improve delay in initiation of treatment

-In order to ensure review intervals were being met, service capacity was increased through implementation of a training programme to involve optometrists in the assessment of patients

- In order to improve appointment times, it was necessary to increase treating capacity of the MTC. With several research trials taking place that had strict protocols, often patients in mainstream macula clinic were given less priority when appointments were made. In addition to this the number of patients requiring monthly follow-up visits was growing and the service was overwhelmed in its ability to treat AMD effectively. Thus it was imperative to find a way to expand the dedicated staff to satisfy all the monitoring and treatment needs in the MTC. Medical workforce was increased by employing more medical retina consultants and medical retina fellows. Increased medical staff allowed provision for up to 10 sessions a week and ad-hoc evening sessions that were dedicated to the management of AMD patients only. Originally there were:

\section{3 medical retina consultants}

2 ophthalmic fellows

1 specialist nurse,

1 optometrist and

1 imaging technician

By the end of our investigation period there were:

4 medical retina consultants,

2 vitreo-retinal consultants,

4 medical retina fellows

2 vitreo-retinal fellows and

2 associate specialists providing service in MTC

-Treating capacity was also increased by increasing the number of rooms from 2 to 3 where patients were assessed prior to injection.

- The required standards were monitored within the department and centrally by including aspects of MTC work in the central trust clinical activity dashboard

- Regular consultant meetings were initiated regarding outstanding issues of MTC 
not be fully representative of routine clinical practice.

\section{Results}

The changes made to improve the treatment of AMD patients has proved successful as seen by the re-audit figures and experienced subjectively by the treating ophthalmologists.

Our results show that $88 \%$ of the treated patients maintained vision in 2011 compared to $79 \%$ in the previous year. Similarly, the number of patients gaining greater than 15 ETDRS letters rose to $20 \%$ in 2011 compared to only $6 \%$ the previous year. Mean visual acuity improved at the end of one year in landmark studies by $7-11$ letters. Our results showed an improvement from mean 3.69 letters loss in 2010 to 2.72 letters gain in 2011.

These visual outcome changes clearly correlate with the reduction in the interval between appointments. Referral to 1 st assessment time was within 1 week for $60 \%$ of the patients in 2011 compared to $28 \%$ in 2009. The time interval between treatment decision to $1 \mathrm{st}$ treatment was a mean of 15 days in 2011 compared to a mean of 70 days in 2009 .

Treatment decision to first treatment was a mean of 15 days in 2011, showing an improvement from 70 days in 2009.

Changes made to the appointment system by improved scheduling and added capacity of the MTC helped to improve vision of patients.

The visual improvement can be demonstrated by higher number of patients gaining significant vision as a result of their therapy as well as fewer patients losing significant vision.

All of these changes not only directly increased treating capacity, but they allowed stronger teams to form in a better organized environment that contributed to better staff morale and work satisfaction which also resulted in better patient satisfaction.

See supplementary file: ds1904.docx - "Results"

\section{Lessons and Limitations}

Results show changes that were made to the service provided at the MTC resulted in improved visual acuity. These changes aimed to reduce the time interval between appointments to reach targets set by the Royal College of Ophthalmologists.

Our study showed that complying with the recommended time intervals during the loading phase of the treatment course, in itself, improved the visual outcome. The visual acuity measures are still poorer than in those found in previous studies. It can be speculated that the difference can be attributed to the less frequent injections based on as needed treatment protocol. However, there are other factors to consider including the progression of the disease and irreversible pathological changes in the eye often documented at first presentation. The sample population of a clinical trial may also
The reduced visual outcome results in 2010 correlate with the poor adherence to timely treatment and a increased time to first treatment. The majority of the changes that were made between 2009-2011 were implemented after the 2010 audit. The results seen in 2010 were rectified by 2011 and the effects of the changes made the MTC are reflected by the improved visual acuity and adherence to appointment standards.

One challenge faced was that in its present form delivery of treatment for wet AMD is costly and labour intensive. Standard outpatient tariffs do not adequately reflect the resource required. The cost of treatment was funded through a service level agreement and commissioners were charged a cost which factored in the staffing required, cost of drug, maintenance and overheads of the imaging equipment, consulting rooms and dedicated treatment room. The department of health has since introduced PBR tariffs for delivery of intravitreal therapy which reflect these requirements. In future costs may be streamlined by reviewing stable patients in separate virtual clinics or monitoring in the community through collaboration with optometrists.

\section{Conclusion}

Our study showed that complying with the recommended time intervals during the loading phase of the treatment of wet AMD, in itself, improved the visual outcome. The poorer visual acuity measures than in those found in previous studies can be attributed to the less frequent injections based on as needed treatment protocol and irreversible pathological changes in the eye often documented at first presentation. The sample population of a clinical trial may also not be fully representative of the one that we face to treat. The reduced visual outcome results in 2010 correlate with the poor adherence to timely treatment. The majority of the changes were implemented after the 2010 audit. The 2011 audit revealed the effects of the changes made that was reflected by the improved visual acuity and adherence to appointment standards.

It is important to continue the audit spiral to ensure effects of the changes made at the MTC are sustained and standards are constantly being improved. Since the last audit, infrastructure was further expanded with extra rooms added to the MTC. Optometrists added to capacity to assess patients under consultant supervision. Virtual clinics were set-up whereby patients attend an assessment appointment and only those come for injection whose results reviewed by an assessing ophthalmologist indicate the necessity of treatment. This increases capacity as the assessment sessions don't need to be staffed by doctors and a higher volume of patients can be reviewed per session by a doctor. This concept seems to be improving follow-up times as consultants are able to go through many patient assessments quickly in one session and identify those patients that need to come in again for treatment. Electronic patient records implementation is expected to reduce appointment time increasing capacity further by making documentation and review of history quicker allowing more time to spend with direct patient care in clinic. 
To further improve our patient related results continuous prospective data collection is planned using electronic patient record to be able to compare our results to other groups'.

\section{References}

1. Owen CG, Fletcher AE, Donoghue M, Rudnicka AR. How big is the burden of visual loss caused by age related macular degeneration in the United Kingdom? $\mathrm{Br} \mathrm{J}$ Ophthalmol. 2003 Mar;87(3):312-7.

2. National Institute for Health and Clinical Excellence. Ranibizumab and Pegaptanib for the treatment of agerelated macular degeneration: NICE technology appraisal guidance 155. [Online: pdf] 2008. Available from: http://www.nice.org.uk/nicemedia/live/12057/41719/41719.p df [Accessed: 23rd Mar 2013].

3. Mitchell P, Korobelnik JF, Lanzetta P, Holz FG, Prunte C, Schmidt-Erfurth $U$, et al. Ranibizumab (Lucentis) in neovascular age-related macular degeneration: evidence from clinical trials. Br J Ophthalmol 2010 Jan;94(1):2-13.

4. Cohen SY, Mimoun G, Oubraham H, Zourdani A, Malbrel $\mathrm{C}$, Quere S, et al. Changes in visual acuity in patients with wet age-related macular degeneration treated with intravitreal ranibizumab in daily clinical practice: The LUMIERE Study. Retina 2013 Mar;33(3):474-481.

5. The Royal College of Ophthalmologists. Age-Related Macular Degeneration Guidelines for Management. [Online: pdf] 2009. Available from:

http://www.rcophth.ac.uk/page.asp?section=451\&sectionTitl $\mathrm{e}=$ Clinical+Guidelines [Accessed: 23rd Mar 2013].

6. Arias L, Armada F, Donate $\mathrm{J}$ et al. Delay in treating agerelated macular degeneration in Spain is associated with progressive vision loss. Eye 2009;23:326-333

7. Shona O, Gupta B, Vemala R, Sivaprasad S. Visual acuity outcomes in ranibizumab-treated neovascular age-related macular degeneration; stratified by baseline vision. Clin Experiment Ophthalmol 2011 Jan;39(1):5-8.

8. Brown DM, Kaiser PK, Michels M et al. Ranibizumab versus verteporfin for neovascular age-related macular degeneration. N Engl J Med 2006;355:1432-1444.

9. Rosenfeld PJ, Brown DM, Heier JS et al. Ranibizumab for neovascular age-related macular degeneration. N Engl J Med 2006;355:1419-1431.

10. Regillo CD, Brown DM, Abraham $P$ et al. Randomized, double-masked, shamcontrolled trial of ranibizumab for neovascular age-related macular degeneration: PIER Study year 1. Am J Ophthalmol 2008;145:239-248.

11. Fung AE, Lalwani GA, Rosenfeld PJ et al. An optical coherence tomography-guided, variable dosing regimen with intravitreal ranibizumab (Lucentis) for neovascular agerelated macular degeneration. Am J Ophthalmol 2007;143:566-583.

\section{Declaration of interests}

Nothing to declare

\section{Acknowledgements}

None 